\title{
Optimal coding and the origins of Zipfian laws
}

\author{
Ramon Ferrer-i-Cancho ${ }^{\mathrm{a}}$, Christian Bentz ${ }^{\mathrm{b}, \mathrm{c}}$ and Caio Seguin ${ }^{\mathrm{d}}$ \\ ${ }^{a}$ Complexity \& Quantitative Linguistics Lab, LARCA Research Group, Departament de \\ Ciències de la Computació, Universitat Politècnica de Catalunya, Campus Nord, Edifici \\ Omega, Jordi Girona Salgado 1-3. 08034 Barcelona, Catalonia (Spain). ORCiD: \\ 0000-0002-7820-923X.rferrericancho@cs.upc.edu. \\ ${ }^{\mathrm{b}}$ URPP Language and Space, University of Zürich, Freiestrasse 16, CH-8032 Zürich, \\ Switzerland. ORCiD: 0000-0001-6570-9326. chris@christianbentz.de \\ 'DFG Center for Advanced Studies "Words, Bones, Genes, Tools", University of Tübingen, \\ Rümelinstraße 23, D-72070 Tübingen, Germany. ORCiD: 0000-0001-6570-9326. \\ ${ }^{\mathrm{d}}$ Melbourne Neuropsychiatry Centre, The University of Melbourne and Melbourne Health, \\ Melbourne, VIC 3010, Australia. ORCiD: 0000-0001-9384-6336. caio.seguin@unimelb.edu.au.
}

\section{ARTICLE HISTORY}

Compiled June 1, 2020

\begin{abstract}
The problem of compression in standard information theory consists of assigning codes as short as possible to numbers. Here we consider the problem of optimal coding - under an arbitrary coding scheme - and show that it predicts Zipf's law of abbreviation, namely a tendency in natural languages for more frequent words to be shorter. We apply this result to investigate optimal coding also under so-called nonsingular coding, a scheme where unique segmentation is not warranted but codes stand for a distinct number. Optimal non-singular coding predicts that the length of a word should grow approximately as the logarithm of its frequency rank, which is again consistent with Zipf's law of abbreviation. Optimal non-singular coding in combination with the maximum entropy principle also predicts Zipf's rank-frequency distribution. Furthermore, our findings on optimal non-singular coding challenge common beliefs about random typing. It turns out that random typing is in fact an optimal coding process, in stark contrast with the common assumption that it is detached from cost cutting considerations. Finally, we discuss the implications of optimal coding for the construction of a compact theory of Zipfian laws more generally as well as other linguistic laws.
\end{abstract}

Keywords: Zipf's law for word frequencies, Zipf's law of abbreviation, optimal coding, maximum entropy principle

\section{Introduction}

Zipf's law of abbreviation states that more frequent words tend to be shorter (Zipf, 1949). Its widespread presence in human languages (Bentz \& Ferrer-i-Cancho, 2016), and the growing evidence in other species (Demartsev et al., 2019 ; Favaro et al., 2020, Ferrer-i-Cancho \& Hernández-Fernández, 2013 Ferrer-i-Cancho, HernándezFernández, et al., 2013; Ferrer-i-Cancho \& Lusseau, 2009; Ficken, Hailman, \& Ficken, 
1978: Hailman, Ficken, \& Ficken, 1985, Heesen, Hobaiter, Ferrer-i-Cancho, \& Semple, 2019, Huang, Ma, Ma, Garber, \& Fan, 2020; Luo et al., 2013), calls for a theoretical explanation. The law of abbreviation has been interpreted as a manifestation of compression (Ferrer-i-Cancho, Hernández-Fernández, et al., 2013), assigning strings as short as possible to represent information, a fundamental problem in information theory, and coding theory in particular (Cover \& Thomas, 2006). Here we aim to investigate compression as a fundamental principle for the construction of a compact theory of linguistic patterns in natural communication systems (Ferrer-i-Cancho, 2018). We explore the relationship between compression and Zipf's law of abbreviation, as well as other regularities such as Zipf's law for word frequencies. The latter states that $p_{i}$, the probability of $i$-th most frequent word, follows (Zipf, 1949),

$$
p_{i} \approx i^{-\alpha}
$$

where $\alpha$ is the exponent (a parameter of the distribution) that is assumed to be about 1 (Ferrer-i-Cancho, 2005b). Zipf (1949) referred to Equation 1 as the rank-frequency distribution.

In standard information theory, codes are strings of symbols from a certain alphabet of size $N$ which are used to represent discrete values from a set of $V$ elements, e.g., natural numbers (Borda, 2011). Suppose that the codes have minimum length $l_{\text {min }}$ (with $l_{\min }=1$ by default). For example, if the alphabet is formed by letters $a$ and $b$, the possible codes are

$$
a, b, a a, a b, b a, b b, a a a, a a b, a b a, a b b, b a a, \ldots
$$

As a set of discrete values one may have natural numbers,

$$
1,2,3,4,5,6,7,8,9,10,11, \ldots
$$

For simplicity, we assume that we wish to code for natural numbers from 1 to $V$. These numbers should be interpreted as what one wishes to code for or as indices or identifiers of what one actually wishes to code for. Therefore, if one wished to code for $V$ different objects that are not numbers from 1 to $V$, one should label each object with a distinct number from 1 to $V$.

In that framework, the problem of compression consists of assigning codes to natural numbers from 1 to $V$ in a way to minimize the mean length of the codes, defined as (Cover \& Thomas, 2006)

$$
L=\sum_{i=1}^{V} p_{i} l_{i}
$$

where $p_{i}$ is the probability of the $i$-th number and $l_{i}$ is the length of its code in symbols. The standard problem of compression consists of minimizing $L$ with the $p_{i}$ 's as a given, and under some coding scheme (Cover \& Thomas, 2006). Roughly speaking, a coding scheme is a constraint on how to translate a number into a code in order to warrant successful decoding, namely retrieving the original number from the code from the receiver's perspective. In the examples of coding that will follow, we assume that one wishes to code numbers from 1 to 6 on strings from an alphabet of two letters $a$ and $b$. Table 1 shows an example of unconstrained coding (no scheme is used). The coding 
Table 1. An example of optimal unconstrained coding of numbers from 1 to 6 on strings from an alphabet of two letters $a$ and $b$.

\begin{tabular}{rr} 
Number & Code \\
\hline 1 & $a$ \\
2 & $a$ \\
3 & $a$ \\
4 & $b$ \\
5 & $b$ \\
6 & $b$
\end{tabular}

Table 2. An example of non-singular coding of numbers from 1 to 6 on strings from an alphabet of two letters $a$ and $b$.

\begin{tabular}{rr} 
Number & Code \\
\hline 1 & $a a$ \\
2 & $a b$ \\
3 & $a$ \\
4 & $b$ \\
5 & $b a$ \\
6 & $b b$
\end{tabular}

in that example is optimal because all strings have minimum length but it is not very useful because each string has three numbers as possible interpretations.

Table 2 shows an example of so-called non-singular coding, meaning that a unique code is assigned to each number. Thus, every code has only one possible interpretation. If we assigned the string $a a$ to more than one number, the coding would not be nonsingular. The example in Table 1 is not non-singular either. In the standard problem of compression, the alphabet is also a given. Therefore, $L$ is minimized with $N$ constant.

The problem of compression can be related to human languages in two ways: either we think of the numbers as representing word types (distinct words), or as representing meaning types (distinct meanings). In the former case, codes stand for distinct word types, in the latter case, they stand for distinct meanings. If numbers represent word types, then a typical application is to solve the problem of optimal recoding, namely reducing the length of words as much as possible without losing their distinctiveness. If we consider numbers to represent meaning types, then human languages do not perfectly fit the non-singular coding scheme due to polysemy (the same word types can have more than one meaning). However, non-singularity is convenient for language a priori because it reduces the cost of communication from the listeners perspective (Piantadosi, Tilly, \& Gibson, 2012, Zipf, 1949) as well as the cost of vocabulary learning in children (Casas, Català, Ferrer-i-Cancho, Hernández-Fernández, \& Baixeries, 2018). Optimization pressures in both ways - shortening of codes, on the one hand, and reducing polysemy (eventually leading to non-singular coding), on the other - are likely to coexist in real languages, as suggested by experiments (Kanwal, Smith, Culbertson, \& Kirby, 2017). See Ferrer-i-Cancho (2018, Section 5.2) for a possible formalization based on a generalization of standard coding theory.

The information theory concepts introduced above have a direct correspondence with popular terms used in research on language optimization. The non-singular scheme implies least effort for the listener, in G. K. Zipf's terms (Zipf, 1949). Zipf's law of abbreviation was explained as the result of combining two pressures (Kanwal et al., 2017): accuracy, i.e. avoiding ambiguity, and efficiency, i.e. using word forms as short as possible. Communicating with maximum accuracy (no ambiguity) is equiva- 


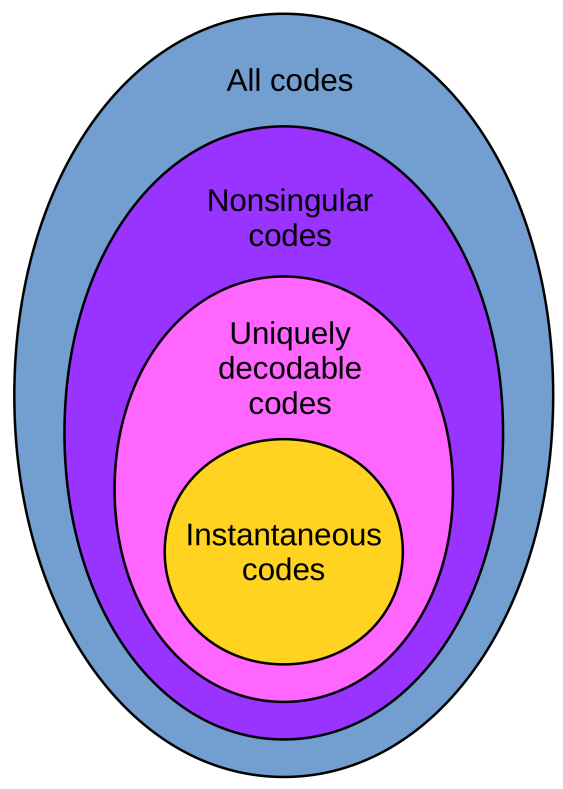

Figure 1. Classes of codes. Adapted from Cover and Thomas (2006, p. 106). Instantaneous codes, that are not described in the main text, are codes such that there is no string in the coding table that matches the beginning of another string (totally or partially). An example of instantaneous code would be the binary representation of numbers from 1 to 6 in the examples of the article.

Table 3. An example of uniquely decodable coding of numbers from 1 to 6 on strings from an alphabet of two letters $a$ and $b$ using Elias gamma encoding (a coding procedure where the code itself tells its length, turning segmentation straightforward (Elias 1975, p. 199).

\begin{tabular}{rr} 
Number & Code \\
\hline 1 & $b$ \\
2 & $a b a$ \\
3 & $a b b$ \\
4 & $a a b a a$ \\
5 & $a a b a b$ \\
6 & $a a b b a$
\end{tabular}

lent to the non-singular scheme. Compression (the minimization of $L$ ) is equivalent to efficiency.

A further coding scheme, which is central to information theory, is uniquely decodable coding, namely, non-singular coding with unique segmentation. That is, when codes are concatenated without a separator, e.g., space, there should be only one way of breaking the sequence into codes. Uniquely decodable codes are hence a subset of non-singular codes (Figure 1).

The coding in Table 2 is not uniquely decodable because the string baba can be interpreted as 4343,55 , etc. In contrast, Table 3 shows a coding that is uniquely decodable. The string baba can here only be interpreted as 12 .

It is easy to see that written English, when written without spaces, is often not uniquely decodable. together can be read as both a single word and also to get her (McMillan, 1956). Godisnowhere illustrates the same problem: it can be read either as God is nowhere or as God is now here. Similar examples can be found in spoken English or other languages. However, unique decodability would be generally convenient for 
Table 4. Optimal non-singular coding of numbers from 1 to 6 on strings consisting of symbols $a$ and $b$. Notice that codes are assigned to frequency ranks.

\begin{tabular}{rr} 
Rank & Code \\
\hline 1 & $a$ \\
2 & $b$ \\
3 & $a a$ \\
4 & $a b$ \\
5 & $b a$ \\
6 & $b b$
\end{tabular}

segmenting speech easily (Romberg \& Saffran, 2010). Again, unique decodability is a listener's requirement, who has to be able to retrieve the codes and the corresponding numbers when the codes are produced in a row (lacking spaces or silences in between them).

Now, suppose that we assign a frequency rank to each number (the most frequent number has rank 1, the 2nd most frequent number has rank 2, and so on). In his pioneering research, Mandelbrot considered the problem of compression implicitly, by assuming that word types are the numbers to code, and wrote that (Mandelbrot, 1966. p. 365) given any prescribed multiset of word probabilities, the average number of letters per word ( $L$ in our notation above) is minimized if the list of words ranked by decreasing probability, coincides with the list of the $V$ shortest letter sequences, ranked by increasing number of letters (as in Table 4 for the case of only two letters). In the language of information theory, he addressed the problem of compression under the scheme of optimal non-singular coding. To our knowledge, a formal proof of the optimality of his coding procedure is still lacking. In fact, information theoretic research has generally neglected the problem of optimal non-singular coding since then, and instead focused on uniquely decodable encoding. The reasons for this are three-fold:

- The primary target of standard information theory are artificial devices (not human brains or natural communication systems).

- The hard segmentation problem arising when non-singular codes are concatenated without separators (word delimiters).

- The waste of time/space when separators are added to facilitate segmentation over these codes (Cover \& Thomas, 2006, p. 105).

These considerations may have prevented information theory from providing simple explanations to linguistic laws.

The remainder of the article is organized as follows. Section 2 presents a generalization of the problem of compression that predicts the law of abbreviation under an arbitrary coding scheme. This type of compression problem is used to prove that non-singular coding consists of assigning a string as short as possible (preserving nonsingularity) to each number following frequency ranks in ascending order - as expected by Mandelbrot (1966). As an example, the coding in Table 4 satisfies this design, while that of Table 3 does not (in the latter, all codes are unnecessarily long from nonsingular coding perspective except for rank 1). In case of optimal non-singular coding, Section 2 shows that $l_{i}$ is an increasing logarithmic function of $i$, the frequency rank when $N>1$, and a linear function of $i$ when $N=1$, giving an exact formula in both cases. This prediction is a particular case of Zipf's law of abbreviation.

The logarithmic relation between length and frequency rank that results from optimal non-singular coding is crucial: it provides a justification for the logarithmic con- 
straint that is needed by the most parsimonious derivation of Zipf's rank-frequency distribution based on the maximum entropy principle (Visser, 2013). For this reason, Section 3 revisits Mandelbrot's derivation of Zipf's distribution combining optimal non-singular coding, and the maximum entropy (maxent) principle (Mandelbrot, 1966). This adds missing perspectives to his original analysis, and illustrates the predictive capacity of optimal non-singular coding with regards to linguistic laws. Although the distribution of word frequencies is power-law-like, an exponential distribution is found for other linguistic units, e.g. part-of-speech tags (Tuzzi, Popescu, \& Altmann, 2010, p. 116-122), colors (Ramscar, 2019), kinship terms (Ramscar, 2019) and verbal alternation classes (Ramscar, 2019). Beyond texts, exponential distributions are found in first names in the census or social security records (Ramscar, 2019). Non-singular coding and maxent can shed light on the emergence of these two types of distributions. In particular, Section 3 shows how the combination of the maximum entropy principle and optimal non-singular coding predicts two different distributions of ranks depending on the value of $N$. When $N>1$, it predicts Equation 1 . When $N=1$, it predicts a geometric distribution of ranks, namely,

$$
p_{i}=q(1-q)^{i-1}
$$

where $q$ is a parameter between 0 and 1 . In addition, such a geometric distribution may arise from suboptimal coding when $N>1$.

Section 4 then challenges the long-standing believe that random typing constitutes evidence that Zipfian laws (Zipf's rank-frequency law and Zipf's law of abbreviation) can be derived without any optimization or cost-cutting consideration (Chaabouni, Kharitonov, Dupoux, \& Baroni, 2019, Kanwal et al., 2017, Li, 1998; Miller, 1957): random typing emerges as an optimal non-singular coding system in disguise. In addition, we investigate various properties of random typing, applying results on optimal coding from Section 2, and providing a simple analytical expression for the relationship between the probability of a word and its rank - a result that Mandelbrot (1966) believed to be impossible to obtain.

Section 5 discusses the implications for empirical research on linguistic laws and how compression, optimal coding and maximum entropy can contribute to the construction of a general but compact theory of linguistic laws.

\section{Optimal coding}

Here we investigate a generalization of the problem of compression, where $L$ (Equation 3 is generalized as mean energetic cost, i.e.

$$
\Lambda=\sum_{i=1}^{V} p_{i} \lambda_{i}
$$

and $p_{i}$ and $\lambda_{i}$ are, respectively, the probability and the energetic cost of the $i$-th type. Without any loss of generality, suppose that the types to be coded are sorted nonincreasingly, i.e.

$$
p_{1} \geq p_{2} \geq \ldots \geq p_{V}
$$


Roughly speaking, a nonincreasing order is the outcome of sorting in decreasing order. We refer to it as nonincreasing instead of decreasing because, strictly, a decreasing order can only be obtained if all the values are distinct.

The generalization is two-fold. First, $\lambda_{i}=g\left(l_{i}\right)$, where $g$ is a strictly monotonically increasing function of $l_{i}$. Second, $l_{i}$ is generalized as a magnitude, namely, a positive real number. When $g\left(l_{i}\right)=l_{i}$ and $l_{i}$ is the length in symbols of the alphabet, $\Lambda$ becomes $L$ (Equation 3), the mean code length of standard information theory (Cover \& Thomas, 2006). The generalization function $g$ follows from other research on the optimization of communication where the energetic cost of the distance between syntactically related words in a linear arrangement is assumed to be a strictly monotonically increasing function of that distance (Ferrer-i-Cancho, 2015). The goal of $g$ is abstracting away from the translation of some magnitude (word length or distance between words) into a real energetic cost. Here we investigate the minimization of $\Lambda$ when the $p_{i}$ 's are constant (given) as in the standard problem of compression, where the magnitudes are lengths of strings following a certain scheme (Cover \& Thomas, 2006).

\subsection{Unconstrained optimal coding}

The solution to the minimization of $\Lambda$ when no further constraint is imposed is that all types have minimum magnitude, i.e.

$$
l_{i}=l_{\min } \text { for } i=1,2, \ldots, V \text {. }
$$

Then $\Lambda$ is minimized absolutely when $l_{\min }=0$, the smallest possible magnitude.

Now suppose that $l_{i}$ is a length as in standard information theory. The condition in Equation 7 implies that all types are assigned the empty string. Then the coding fails to be non-singular (for $V>1$ ). If empty strings are not allowed then $l_{\min }=1$. In that case, optimal coding will produce codes that are not non-singular if $N<V$ (as in Table 1). One may get codes that are non-singular by increasing $N$. However, recall that $N$ is constant in the standard problem of compression.

First, we will investigate the problem of compression (minimization of $\Lambda$ ) when the lengths are generalized to magnitudes (positive real numbers) that belong to a given multiset. Second, we will apply the results to the problem of compression in the non-singular scheme (the multiset contains the lengths of all distinct strings).

\subsection{Optimal coding with given magnitudes}

Suppose that we wish to minimize $\Lambda$ where the $l_{i}$ 's are taken from a multiset $\mathcal{L}$ of real positive values with $|\mathcal{L}| \geq V$. For instance, the values could be the length in symbols of the alphabet or the duration of the type. An assignment of elements of $\mathcal{L}$ to the $l_{i}$ 's consists of sorting the elements of $\mathcal{L}$ forming a sequence and assigning to each $l_{i}$ the $i$-th element of the sequence. For an assignment, only the $V$ first elements of the sequence matter. After an assignment, the $l_{i}$ 's define a subset of $\mathcal{L}$, i.e.

$$
\left\{l_{1}, \ldots, l_{i}, \ldots, l_{V}\right\} \subseteq \mathcal{L} .
$$

Therefore, $\mathcal{L}$ is a given in addition to the $p_{i}$ 's. $\mathcal{L}$ allows one to capture arbitrary constraints on word length, beyond the traditional coding schemes (e.g., non-singular 
coding or uniquely decodable encoding). Perceptibility and distinguishability factors may prevent the use of very short strings, even under a uniquely decodable scheme. Phonotactics (a branch of phonology) shows that not all possible combinations of phonemes are present in a language. Certain phonemes or combinations are harder (if not impossible) to articulate or perceive. See Akmajian, Demers, Farmer, and Harnish (1995, chapters 3 and 4) for an overview of these concepts and constraints from linguistics.

This problem of compression is more general than the compression problem in standard information theory because:

- $l_{i}$ is generalized as a magnitude, namely a positive real number. The strings, even when the magnitude is a length, are irrelevant.

- In case the magnitudes are string lengths, the non-singular coding scheme is obtained defining $\mathcal{L}$ as the lengths of all the different strings that can be formed. Similarly, in case of uniquely decodable coding, the string lengths have to allow one to find strings that produce them while preserving the constraints of the scheme.

These two generalization allow us to shed light on the origins of Zipf's law of abbreviation in human languages, where words do not match perfectly the constraints of traditional schemes, as well as in other species, where the coding scheme is unknown and the magnitude is measured as a time duration, namely a positive real value (e.g., Heesen et al. (2019); Semple, Hsu, and Agoramoorthy (2010)). Moreover, it is conceivable that certain natural communication systems do not build signs by combining elementary units (such as phonemes or syllables as in human languages) - as assumed by standard information theory - but rather hollistically. Such cases could be implemented as strings of length 1 and their magnitude could be a real number indicating their expected duration.

When $|\mathcal{L}|=V$, there are as many different assignments as different sequences that can be produced from $\mathcal{L}$. When $|\mathcal{L}| \geq V$, the solution to the problem of compression consists of finding $\Lambda_{\text {min }}$, the minimum value of $\Lambda$, and the assignments that achieve the minimum, over all the

$$
\frac{|\mathcal{L}| !}{(|\mathcal{L}|-V) !}
$$

assignments of elements of $\mathcal{L}$ to the $l_{i}$ 's. We will show that $\Lambda$ is minimized exclusively by all the assignments from orderings of the elements of $\mathcal{L}$ such that the $V$ first elements are the $V$ smallest elements of $\mathcal{L}$ sorted in nondecreasing order (we refer to it as nondecreasing instead of increasing because, strictly, an increasing order can only be obtained if all the values are distinct). There is only one assignment if the values in $\mathcal{L}$ are distinct and $|\mathcal{L}|=V$.

Suppose that $n_{c}$ is the number of concordant pairs of an assignment. $\left(p_{i}, l_{i}\right)$ and $\left(p_{j}, l_{j}\right)$ are said to be concordant if

$$
\operatorname{sgn}\left(p_{i}-p_{j}\right) \operatorname{sgn}\left(l_{i}-l_{j}\right)=1,
$$

where sgn is the sign function, i.e.

$$
\operatorname{sgn}(x)=\left\{\begin{array}{c}
\frac{x}{|x|} \text { if } x \neq 0 \\
0 \text { if } x=0 .
\end{array}\right.
$$


Equation 8 is equivalent to

$$
\operatorname{sgn}\left(p_{i}-p_{j}\right)=\operatorname{sgn}\left(l_{i}-l_{j}\right) \neq 0
$$

The following lemma gives a crucial necessary condition of optimal configurations:

Lemma 2.1. $\Lambda=\Lambda_{\text {min }}$ implies that the sequence $l_{1}, \ldots, l_{i}, \ldots, l_{V}$ is sorted in nondecreasing order, i.e. $n_{c}=0$ over

$$
\left(p_{1}, l_{1}\right), \ldots,\left(p_{i}, l_{i}\right), \ldots\left(p_{V}, l_{V}\right)
$$

because the sequence $p_{1}, \ldots, p_{i}, \ldots, p_{V}$ is sorted in nonincreasing order.

Proof. We will proof the contrapositive, namely that $n_{c}>0$ implies $\Lambda>\Lambda_{\text {min }}$ adapting arguments in previous work (Ferrer-i-Cancho, Hernández-Fernández, et al., 2013). Let the pair $\left(p_{i}, l_{i}\right)$ and $\left(p_{j}, l_{j}\right)$ be concordant (then $i \neq j$ ) and such that $1 \leq i, j \leq V$. Without any loss of generality, suppose that $i<j$. Then $p_{i}>p_{j}$ by Equation 6 (the case $p_{i}=p_{j}$ is excluded as the pair is concordant) and $l_{i}>l_{j}$ because the pair is concordant. If we swap $l_{i}$ and $l_{j}$, then $\Lambda$ will become

$$
\begin{aligned}
\Lambda^{\prime} & =\Lambda-p_{i} \lambda_{i}-p_{j} \lambda_{j}+p_{i} \lambda_{j}+p_{j} \lambda_{i} \\
& =\Lambda+\left(p_{i}-p_{j}\right)\left(\lambda_{j}-\lambda_{i}\right)
\end{aligned}
$$

and then the difference between the final and the initial value of $\Lambda$ becomes

$$
\begin{aligned}
\Delta & =\Lambda^{\prime}-\Lambda \\
& =\left(p_{i}-p_{j}\right)\left(\lambda_{j}-\lambda_{i}\right) .
\end{aligned}
$$

It is easy to see that $\Lambda>\Lambda_{\min }$ as we wished because $\Delta<0$. Recall that, in this context, $p_{i}>p_{j}$ and $l_{i}>l_{j}$ (as explained above) and that $g$ is a strictly monotonically increasing function (notice that also $\Delta<0$ when $1 \leq j<i \leq V$ ).

An assignment stemming from sorting the $V$ smallest elements of $\mathcal{L}$ in nondecreasing order (increasing order if the $V$ smallest elements of $\mathcal{L}$ are distinct) is equivalent to one where $n_{c}=0$. The following theorem expresses it formally:

Theorem 2.2. $\Lambda=\Lambda_{\text {min }}$ if and only if two conditions are met

1. $l_{1}, \ldots, l_{i}, \ldots, l_{V}$ are the $V$ smallest elements of $\mathcal{L}$.

2. The sequence $l_{1}, \ldots, l_{i}, \ldots, l_{V}$ is sorted in nondecreasing order, i.e. $n_{c}=0$ over

$$
\left(p_{1}, l_{1}\right), \ldots,\left(p_{i}, l_{i}\right), \ldots\left(p_{V}, l_{V}\right)
$$

because the sequence $p_{1}, \ldots, p_{i}, \ldots, p_{V}$ is sorted in nonincreasing order.

Proof. We proceed proving each direction of the equivalence separately.

(1) $\Lambda=\Lambda_{\min }$ implies conditions 1 and 2

We will prove the contrapositive, namely that the failure of condition 1 or 2 implies $\Lambda>\Lambda_{\text {min }}$. 
(a) Suppose that condition 1 fails. Then there is an element $l^{\prime}$ in $\mathcal{L} \backslash$ $\left\{l_{1}, \ldots, l_{i}, \ldots, l_{V}\right\}$ such that $l^{\prime}<\max \left(l_{1}, \ldots, l_{i}, \ldots, l_{V}\right)$, where $\backslash$ is the multiset difference operator. Suppose that $k$ is the index of a magnitude such that $1 \leq k \leq V$ and $l_{k}>l^{\prime}$. Assigning $l^{\prime}$ to $l_{i}, \Lambda$ will decrease strictly because $l_{k}>l^{\prime}$. Thus, the original value of $\Lambda$ satisfied $\Lambda>\Lambda_{\text {min }}$.

(b) Suppose that condition 2 fails. Then $\Lambda>\Lambda_{\min }$ by the contrapositive of Lemma 2.1.

(2) Conditions 1 and 2 imply $\Lambda=\Lambda_{\min }$

We will show the contrapositive, namely that $\Lambda>\Lambda_{\text {min }}$ implies that condition 1 or 2 fails. $\Lambda>\Lambda_{\min }$ can happen when condition 1 fails, as we have seen above. Suppose that condition 1 does not fail. Can we conclude that condition 2 fails? Let $l_{i}^{\text {min }}$ and $\lambda_{i}^{\text {min }}$ be the values of $l_{i}$ and $\lambda_{i}$, respectively, in some minimum assignment, namely one yielding $\Lambda=\Lambda_{m i n}$. By Lemma 2.1, the sequence $l_{1}^{\min }, \ldots, l_{i}^{\min }, \ldots, l_{V}^{\min }$ is sorted in nondecreasing order and its corresponding number of concordant pairs is $n_{c}^{\text {min }}=0$. Notice that $\Lambda>\Lambda_{\min }$ implies that the $V$ smallest values of $\mathcal{L}$ are not identical (otherwise $\Lambda=\Lambda_{\min }$ for any assignment satisfying condition 1). With this clarification in mind, it is easy to see that there must be some $i$ such that $\lambda_{i}>\lambda_{i}^{\text {min }}$, or equivalently, $l_{i}>l_{i}^{\text {min }}$. If that did not happen, then one would have $\lambda_{j} \leq \lambda_{j}^{\text {min }}$ for each $j$ such that $1 \leq j \leq V$ and then $\Lambda \leq \Lambda_{\min }$, contradicting $\Lambda>\Lambda_{\min }$. Crucially, such particular $i$ prevents the $l_{i}$ 's from having the non-decreasing order that is defined by the $l_{i}^{\text {min }}$ 's, leading to $n_{c}>0$ by condition 1 and $n_{c}^{\text {min }}=0$, as we wished.

The Kendall $\tau$ correlation between the $p_{i}$ 's and the $l_{i}$ 's is (Conover, 1999)

$$
\tau\left(p_{i}, l_{i}\right)=\frac{n_{c}-n_{d}}{\left(\begin{array}{c}
V \\
2
\end{array}\right)}
$$

where $n_{d}$ is the number of discordant pairs. $\left(p_{i}, l_{i}\right)$ and $\left(p_{j}, l_{j}\right)$ are said to be discordant if

$$
\operatorname{sgn}\left(p_{i}-p_{j}\right) \operatorname{sgn}\left(l_{i}-l_{j}\right)=-1 \text {. }
$$

or, equivalently,

$$
\operatorname{sgn}\left(p_{i}-p_{j}\right)=-\operatorname{sgn}\left(l_{i}-l_{j}\right) \neq 0
$$

In our context,

$$
\tau\left(p_{i}, l_{i}\right)=\frac{1}{\left(\begin{array}{c}
V \\
2
\end{array}\right)} \sum_{i<j} \operatorname{sgn}\left(p_{i}-p_{j}\right) \operatorname{sgn}\left(l_{i}-l_{j}\right) .
$$

An implication of optimal coding (minimum $\Lambda$ ) is that $\tau\left(p_{i}, l_{i}\right)$ cannot be positive. Formally:

Corollary 2.3. $\Lambda=\Lambda_{\text {min }}$ implies $\tau\left(p_{i}, l_{i}\right) \leq 0$ with equality if and only if $n_{d}=0$. 
Proof. By Lemma $2.1 \Lambda=\Lambda_{\min }$ implies $n_{c}=0$ and then

$$
\tau\left(p_{i}, l_{i}\right)=-\frac{n_{d}}{\left(\begin{array}{c}
V \\
2
\end{array}\right)}
$$

Since $n_{d} \geq 0$ one has $\tau\left(p_{i}, l_{i}\right) \leq 0$, with equality if and only if $n_{d}=0$.

\subsection{Optimal non-singular coding}

Under the scheme of uniquely decodable codes, standard information theory tells us that the minimization of $L$ leads to (Cover \& Thomas, 2006)

$$
l_{i} \propto\left\lceil-\log _{N} p_{i}\right\rceil
$$

which is indeed a particular case of Zipf's law of abbreviation. This corresponds to the minimization of $\Lambda$ with $g$ as the identity function in our framework. Here we wish to minimize $\Lambda$ with $l_{i}$ as the length of the $i$-th most frequent type when only the $p_{i}$ 's are prescribed under the non-singular coding scheme (Figure 1).

Under non-singular coding, the set of available strings consists of all the different strings of symbols that can be built with an alphabet of size $N$. There are $N^{l}$ different strings of length $l$. Let $S$ be the infinite sequence of these strings sorted by increasing length (the relative ordering of strings of the same length is arbitrary). If empty strings are not allowed, the strings in positions 1 to $N$ have length 1 , the strings in positions $N+1$ to $N+N^{2}$ have length 2 , and so on as in 2 for $N=2$.

Corollary 2.4. Optimal non-singular coding consists of assigning the $i$-th string of $S$ to the $i$-th most probable type for $1 \leq i \leq V$.

Proof. We define $\mathcal{L}$ as the multiset of the lengths of the strings in $S$. As there is a oneto-one correspondence between an element of $\mathcal{L}$ and an available string, the application of theorem 2.2 with $g$ as the identity function gives that the optimal coding is such that

- The sequence $l_{1}, \ldots, l_{i}, \ldots, l_{V}$ contains the $V$ smallest lengths, and then comprises the codes that are the shortest possible strings.

- $l_{1}, \ldots, l_{i}, \ldots, l_{V}$ is sorted in nondecreasing order, and then the $i$-th type is assigned the $i$-th shortest string.

\subsection{Length as a function of frequency rank in optimal non-singular coding}

We aim to derive the relationship between the rank of a type (defined according to its probability) and its length in case of optimal non-singular codes for $N \geq 1$. Suppose that $p_{i}$ is the probability of the $i$-th most probable type and that $l_{i}$ is its length. The following lemma addresses a generalization of the problem:

Lemma 2.5. If rank $i$ is assigned the shortest possible string that has length $l_{\text {min }}$ or 
greater then

$$
l_{i}= \begin{cases}\left\lceil\log _{N}\left((1-1 / N) i+N^{l_{\text {min }}-1}\right)\right\rceil & \text { for } N>1 \\ i+l_{\text {min }}-1 & \text { for } N=1 .\end{cases}
$$

Proof. Then the largest rank of types of length $l$ is

$$
i=\sum_{k=l_{\min }}^{l} N^{k}
$$

When $N>1$, we get

$$
i=\frac{N^{l+1}-N^{l_{\min }}}{N-1}
$$

and equivalently

$$
N^{l}=\frac{1}{N}\left[(N-1) i+N^{l_{m i n}}\right]
$$

Taking logs on both sides of the equality, one obtains

$$
l=\frac{\log \left(\frac{1}{N}\left[(N-1) i+N^{l_{\text {min }}}\right]\right)}{\log N} .
$$

The result can be generalized to any rank of types of length $l$ as

$$
l=\left\lceil\frac{\log \left(\frac{1}{N}\left[(N-1) i+N^{l_{\text {min }}}\right]\right)}{\log N}\right\rceil .
$$

Changing the base of the logarithm to $N$, one obtains

$$
l=\left\lceil\log _{N}\left((1-1 / N) i+N^{l_{\text {min }}-1}\right)\right\rceil .
$$

Alternatively, Equation 11 also yields

$$
\begin{aligned}
l & =\left\lceil\frac{\log \left[(N-1) i+N^{l_{\text {min }}}\right]}{\log N}-1\right\rceil \\
& =\left\lceil\log _{N}\left[(N-1) i+N^{l_{\text {min }}}\right]\right\rceil-1
\end{aligned}
$$

The case $N=1$ is trivial, one has $l=i+l_{\min }-1$. Therefore, the length of the $i$-th most probable type follows Equation 10 .

The previous arguments allow one to conclude:

Corollary 2.6. In case of optimal coding with non-singular codes, the length of the $i$-th most probable type follows Equation 10 with $l_{\text {min }}=1$. 
When $N>1$, one obtains

$$
l_{i}=\left\lceil\log _{N}((1-1 / N) i+1)\right\rceil,
$$

the same conclusion was reached by Sudan $(2006)$ though lacking a detailed explanation.

\subsection{Relationships with other mathematical problems}

We have investigated a problem of optimal coding where magnitudes stem from a given multiset of values. The problem is related to other mathematical problems outside coding theory. Notice that $\Lambda$ can be seen as a scalar product of two vectors, i.e. $\vec{p}=\left\{p_{1}, \ldots, p_{i}, \ldots, p_{V}\right\}$ and $\vec{\lambda}=\left\{\lambda_{1}, \ldots, \lambda_{i}, \ldots, \lambda_{V}\right\}$ and $L$ as a scalar product of $\vec{p}$ and $\vec{l}=\left\{l_{1}, \ldots, l_{i}, \ldots, l_{V}\right\}$. When $|\mathcal{L}|=V$ the problem is equivalent to minimizing the scalar (or dot) product of two vectors (of positive real values) over all the permutations of the content of each vector (Aadam, 2016). By the same token, the problem is equivalent to minimizing the Pearson correlation between $\vec{p}$ and $\vec{\lambda}$ when the content (but not the order) of each vector is preserved. Recall that the Pearson correlation between $\vec{p}$ and $\vec{\lambda}$ can be defined as Conover, 1999)

$$
r(\vec{p}, \vec{\lambda})=\frac{\vec{p} \cdot \vec{\lambda}-\mu_{p} \mu_{\lambda}}{\sigma_{p} \sigma_{\lambda}},
$$

where $\mu_{x}$ and $\sigma_{x}$ are, respectively, the mean and the standard deviation of vector $\vec{x}$.

The link with Pearson correlation goes back to the original coding problem: such a correlation has been used to find a concordance with the law of abbreviation that is in turn interpreted as a sign of efficient coding (Semple et al. 2010). According to Equation 12, such a correlation turns out to be a linear transformation of the cost function. Put differently, minimizing $\Lambda$ with prescribed $p_{i}$ 's and with $\lambda_{i}$ as the identity function (as it is customary in standard coding theory), is equivalent to minimizing the Pearson correlation at constant mean and standard deviation of both probabilities and magnitudes. Therefore, the Pearson correlation is a measure of the degree of optimization of a system when these means and standard deviations are constant (it is implicit that the standard deviations are not zero, otherwise the Pearson correlation is not defined).

\section{The maximum entropy principle}

Now we turn onto the question of making a safe prediction on the distribution of word ranks in case of optimal non-singular coding. The maximum entropy principle states that Kesavan, 2009)

Out of all probability distributions consistent with a given set of constraints, the distribution with maximum uncertainty should be chosen.

The distribution of word frequencies has been derived via maximum entropy many times with similar if not identical methods (Baek, Bernhardsson, \& Minnhagen, 2011; Ferrer-i-Cancho, 2005a; Liu, 2008; Mandelbrot, 1966; Naranan \& Balasubrah- 
manyan, 1992a, 1993; Visser, 2013). Depending on the study, the target was Zipf's rank-frequency distribution, Equation 1. (Liu, 2008; Mandelbrot, 1966; Naranan \& Balasubrahmanyan, 1993) or its sister law with frequency as the random variable (Ferrer-i-Cancho, 2005a; Naranan \& Balasubrahmanyan, 1992a), stating that the $n_{f}$, the number of words of frequency $f$, satisfies approximately

$$
n_{f} \approx f^{-\beta}
$$

with $\beta \approx 2$ (Moreno-Sánchez, Font-Clos, \& Corral, 2016; Zipf, 1949). In some cases, maximum entropy is used as an explanation for the ubiquity of power-law-like distributions, with Zipf's law for word frequencies or its sister as a particular case (Baek et al. 2011; Visser, 2013). For simplicity, here we revisit the essence of the principle focusing on how our results on optimal non-singular coding can be used to derive different rank distributions.

The maximum entropy principle allows one to obtain a distribution that maximizes the entropy of probability ranks, namely,

$$
H=-\sum_{i=1}^{V} p_{i} \log p_{i}
$$

under certain constraints on cost over the $i$ 's and a couple of elementary constraints on the $p_{i}$ 's, i.e. $p_{i} \geq 0$ and

$$
\sum_{i=1}^{V} p_{i}=1 .
$$

See Kapur and Kesavan (1992) and Harremoës and Topsøe (2001) for an overview. For simplicity, we assume a single non-elementary cost constraint, namely $L$, as defined in Equation 3. For simplicity, we assume that $V$ is not finite. See Visser (2013) for an analysis of the case of more than one non-elementary constraint and a comparison of the finite versus infinite case. See Harremoës and Topsøe (2001) for some critical aspects of the traditional application of maximum entropy.

In our simple setup, the method leads to distributions of the form

$$
p_{i}=\frac{e^{-\alpha l_{i}}}{Z}
$$

where $\alpha$ is a Lagrange multiplier and

$$
Z=\sum_{j=1}^{\infty} e^{-\alpha l_{j}}
$$

is the partition function. In case of optimal non-singular coding, we have two cases. If $N>1$ then $l_{i} \approx \log _{N} i$ for sufficiently large $N$ (Equation 10), which transforms Equation 13 into a zeta distribution, i.e.

$$
p_{i}=\frac{1}{Z} i^{-\alpha}
$$


while the partition function becomes

$$
Z=\sum_{j=1}^{\infty} j^{-\alpha},
$$

namely the Riemann zeta function. The zeta distribution is an approximation to Zipf's law for word frequencies.

When $N=1$ then $l_{i}=i$ (Equation 10 with $l_{\min }=1$ ), which transforms Equation 13 into an exponential distribution of word frequencies, i.e.

$$
p_{i}=\frac{1}{Z} e^{-\alpha i}
$$

while

$$
Z=\sum_{j=1}^{\infty} e^{-\alpha i}
$$

Applying the same arguments, it is possible to obtain an exponential distribution via maximum entropy for $N>1$ if $l_{i}=i$. In that case, however, the coding would be non-singular (every type would be coded with a string of distinct length) but would not be optimal. Equation 15 matches the exponential-like distribution that is found for certain linguistic units (Ramscar, 2019; Tuzzi et al., 2010). In sum, this distribution may result, according to the maximum entropy principle, from either optimal or suboptimal coding.

Although Equation 15 is for a discrete random variable, it has the form of the popular exponential distribution for continuous random variables. That equation actually matches the definition of the customary geometric distribution in Equation 4 . To see it, notice that $Z$ is the summation of a geometric series where the first term $a$ and the common factor $r$ are the same, i.e. $a=r=e^{-\alpha}$. Therefore, assuming $|r|<1$, i.e. $\alpha>0$,

$$
\begin{aligned}
Z & =\frac{a}{1-r} \\
& =\frac{e^{-\alpha}}{1-e^{-\alpha}}
\end{aligned}
$$

Then equation 15 can be rewritten equivalently as

$$
p_{i}=\frac{1-e^{-\alpha}}{e^{-\alpha}}\left(e^{-\alpha}\right)^{i} .
$$

The substitution $q=1-e^{-\alpha}$ transforms Equation 16 into the customary definition of a geometric distribution in Equation 4 as we wished.

\section{The optimality of random typing}

The results on optimal coding above allow one to unveil the optimality of typing at random, assuming that the space bar is hit with a certain probability and that let- 
ters are equally likely (Miller, 1957). It has been argued many times that random typing reproduces Zipf's rank-frequency distribution (e.g. Li (1992); Miller (1957); Miller and Chomsky (1963); Suzuki, Tyack, and Buck (2005)). In particular, Miller concluded that the law "can be derived from simple assumptions that do not strain one's credulity (unless the random placement of spaces seems incredible), without appeal to least effort, least cost, maximal information, or any other branch of the calculus of variations. The rule is a simple consequence of those intermittent silences which we imagine to exist between successive words." (Miller, 1957). Similarly, Li (1998) argued that "random typing shows that a random process can mimic a cost-cutting process, but not purposely." A similar view is found in reviews of Zipf's law for word frequencies, where optimization and random typing are considered to be different mechanisms (Mitzenmacher, 2003; Newman, 2005). The view of random typing as detached from cost reduction is also found in research on the origins of Zipf's law of abbreviation (Chaabouni et al., 2019; Kanwal et al., 2017). Leaving aside the problem of the poor fit of random typing to their original target, i.e. the distribution of word frequencies (Ferrer-i-Cancho \& Elvevåg, 2009, Ferrer-i-Cancho \& Gavaldà, 2009), these views are also problematic because random typing and least cost are not really independent issues. We will show it through the eye of the problem of compression.

The optimality of random typing can be seen in two ways. One through recoding, namely replacing each word it produces by another string so as to minimize $L$ under the non-singular coding scheme. The other - indeed equivalent - consists of supposing that random typing is used to code for numbers whose probability matches that of the words produced by random typing. In both cases, we will show that the value of $L$ of a random typing process cannot be reduced and thus it is optimal. Put differently, we will show that there is no non-singular coding system that can do it more efficiently (with a smaller $L$ ) than random typing.

It is easy to see that the strings that random typing produces are optimal according to Corollary 2.4. Recall that the probability of a "word" $w$ of length $l$ in random typing is (Ferrer-i-Cancho \& Gavaldà, 2009, p. 838)

$$
p_{l}(w)=\left(\frac{1-p_{s}}{N}\right)^{l} \frac{p_{s}}{\left(1-p_{s}\right)^{l_{\text {min }}}},
$$

where $l$ is the length of $w, p_{s}$ is the probability of producing the word delimiter (a whitespace), $N$ is the size of the alphabet that the words consist of $(N>0)$ and $l_{\text {min }}$ is the minimum word length $\left(l_{\min } \geq 0\right)$. Hereafter we assume for simplicity that $0<p_{s}<1$. If $p_{s}=0$, strings never end. If $p_{s}=1$, all the strings have length $l_{\text {min }}$ and then random typing has to be analyzed following the arguments for unconstrained optimal coding in Section 2.1.

We will show that after sorting nondecreasingly all possible strings of length at least $l_{\text {min }}$ that can be formed with $N$ letters, the $i$-th most likely type of random typing receives the $i$-shortest string. First, Equation 17 indicates that all words of the same length are equally likely and $p_{l+1}(w) \geq p_{l}(w)$ for $l \geq l_{\min }$ because $p_{s}, l_{\min }$ and $N$ are constants. Therefore, the ranks of words of length $l$ are always larger than those of words of length $l+1$. Keeping this property in mind, words of the same length are assigned an arbitrary rank. Second, $p_{l}(w)>0$ for all the $N^{l}$ different words of length $l$ that can be formed. Therefore, all available strings of a given length are used. The optimality of random typing for $N=2$ and $l_{\min }=1$ can be checked easily in Table 5. The exact relationship between rank and length in random typing will be derived below. 
Table 5. The probability $\left(p_{i}\right)$, the length $\left(l_{i}\right)$ of the $i$-th most frequent string (code) or random typing with $N=2$ and $l_{\min }=1 . l_{i}$ is calculated via Equation 10 with $N=2$ and $l_{\min }=1 . p_{i}$ is calculated applying $l_{\text {min }}=1, N=2$ and $l_{i}$ to Equation 19

\begin{tabular}{rrrr} 
Code & $i$ & $l_{i}$ & $p_{i}$ \\
\hline $\mathrm{a}$ & 1 & 1 & $p_{s} / 2$ \\
$\mathrm{~b}$ & 2 & 1 & $p_{s} / 2$ \\
$\mathrm{aa}$ & 3 & 2 & $\left(1-p_{s}\right) p_{s} / 4$ \\
$\mathrm{ab}$ & 4 & 2 & $\left(1-p_{s}\right) p_{s} / 4$ \\
$\mathrm{ba}$ & 5 & 2 & $\left(1-p_{s}\right) p_{s} / 4$ \\
$\mathrm{bb}$ & 6 & 2 & $\left(1-p_{s}\right) p_{s} / 4$ \\
$\mathrm{aaa}$ & 7 & 2 & $\left(1-p_{s}\right)^{2} p_{s} / 8$ \\
$\ldots$ & $\ldots$ & $\ldots$ & $\ldots$
\end{tabular}

Random typing satisfies a particular version of Zipf's law of abbreviation where the length of a word $(l)$ is a linear function of its probability $(p)$, i.e.

$$
l=a \log p+b
$$

where $a$ and $b$ are constants $(a<0)$. Namely, the probability of a word is determined by its length (the characters constituting the words are irrelevant), Equation 17 allows one to express $l$ as a function of $p(w)$. Rearranging the terms of Equation 17, taking logarithms, and replacing $p(w)$ by $p$, one recovers Equation 18 with

$$
a=\left(\log \frac{1-p_{s}}{N}\right)^{-1}
$$

and

$$
b=a \log \frac{\left(1-p_{s}\right)^{l_{\text {min }}}}{p_{s}}
$$

Does random typing also satisfy Zipf's law for word frequencies (Equation 1)? Mandelbrot was aware "that the relation between rank and probability is given by a step function" for the random typing model we have considered here, but he argued that "such a relation cannot be represented by any simple analytic expression" (Mandelbrot, 1966, p. 364). Knowing that random typing is optimal from the standpoint of non-singular coding it is actually possible to obtain a simple analytic expression for $p_{i}$, the probability that random typing produces a word of rank $i$. Replacing $p_{l}(w)$ by $p_{i}$ and $l$ by $l_{i}$, Equation 17 becomes

$$
p_{i}=\left(\frac{1-p_{s}}{N}\right)^{l_{i}} \frac{p_{s}}{\left(1-p_{s}\right)^{l_{\min }}},
$$

where $l_{i}$ the length of the word of rank $i$ that is given by Equation 10. To our knowledge, this is the first exact Equation for $p_{i}$. In previous research, only approximate expressions for $p_{i}$ have been given (Li, 1992, Mandelbrot, 1966; Miller, 1957; Miller \& Chomsky, 1963). These non-rigorous approximations correspond to the Zipf-Mandelbrot law,

$$
p_{i} \propto(i+b)^{-\alpha},
$$


a)

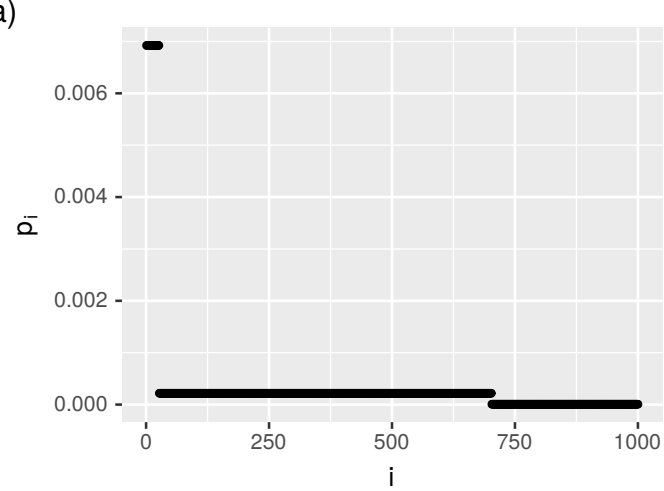

b)

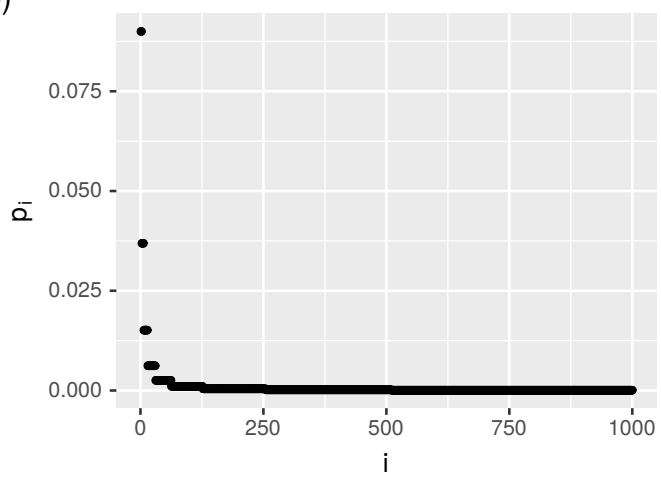

Figure 2. $\quad p_{i}$ the probability of a word of rank $i$ produced by random typing with $p_{s}=0.18, l_{\min }=1$ up to rank $i_{\max }=1000$ (in the standard random typing model, the maximum rank is infinite). a) $N=26$. b) $N=2$.

a generalization of Zipf's law (Equation 1) with an additional parameter $b>0$ (Mandelbrot, 1966), that is actually a smoothed version of Equation 19. If ranks are unbounded as in the random typing model, the Zipf-Mandelbrot law can be defined exactly as

$$
p_{i}=\frac{1}{\zeta(\alpha, b)}(i+b)^{-\alpha}
$$

where

$$
\zeta(\alpha, b)=\sum_{i=0}^{\infty}(i+b)^{-\alpha}
$$

is the Hurwitz zeta function. Equation 21 becomes the definition of the zeta distribution (Equation 14) when $b=1$.

Figure 2 a) shows $p_{i}$ versus $i$ for $N=26$ and $p_{s}=0.18$ applying Equation 19 . These are the parameters that Miller (1957) used in his classic article on random typing to mimic English. The stepwise shape, that is missing in the Zipf-Mandelbrot law (equations 20 and 21), can be smoothed by introducing a bias towards certain letters (Ferrer-i-Cancho \& Elvevåg, 2009, Li, 1992) as in the original setup all letters are equally likely. Reducing $N$ as much as possible will also smooth the shape (reducing $N$ is a particular case of bias that consists of turning 0 the probability of certain symbols). Figure $2 \mathrm{~b}$ ) shows the smoothing effect of $N=2$ (corresponding to the examples given in Table 5). Notice that $N$ cannot be reduced further: we have shown above that $N=1$ transforms the distribution of ranks of random typing into a geometric distribution.

\section{Discussion}

In his pioneering research, Zipf found a tendency of more frequent words to be shorter. He termed this observation the law of abbreviation (Zipf, 1949). However, he never proposed a functional dependency or mathematical model for the relationship between frequency and length.

Here we have filled a gap in standard information theory concerning optimal non- 
singular coding, that predicts $l_{i} \approx \log i$, where $i$ is the probability rank. This result complements the well-known relationship $l_{i} \approx-\log p_{i}$ predicted by optimal uniquely decodable coding (Cover \& Thomas, 2006). Derivations of a logarithmic relationship between the length of a word and its probability rank can be found in classic work (Mandelbrot, 1966, Rapoport, 1982). However, our derivation is novel in the sense of providing a general exact formula (not an approximation; covering $N \geq 1$ and $\left.l_{\min } \geq 0\right)$ and involving optimal non-singular coding in the argument. It was clear to Mandelbrot that "given any prescribed set of word probabilities, the average number of letters per words is minimized if the list of words, ranked by decreasing probability, coincides with the list of the $V$ shortest letter sequences" (Mandelbrot, 1966, p. 365) but he never provided an exact formula for the relationship between $l_{i}$ and $i$ as far as we know. Indeed, he actually thought it was impossible (Mandelbrot, 1966). Likewise, Rapoport did not take information theoretic optimality considerations into account and simply stated that "we shall want the shortest words to be the most frequent" (Rapoport, 1982, p. 9).

Traditionally, quantitative linguistics research has been based on the fit of powerlaw-like models (Sigurd, Eeg-Olofsson, \& van Weijer, 2004; Strauss, Grzybek, \& Altmann, 2007). Surprisingly, the predictions of information theory reviewed above have largely been neglected. The problem concerns not only the relationship between length and frequency but also parallel quantitative linguistics research where frequency is replaced by the frequency rank (see Strauss et al. (2007, p. 274) and references therein). Some notable exceptions are discussed in the following.

In the work by Hammerl (1990), both the relationship $l_{i} \approx \log p_{i}$ and $l_{i} \approx \log i$ are considered. He explains that Guiraud (in 1959) derived $l_{i} \approx \log i$ by "purely combinatorial considerations, where all possible combinations of letters in the respective languages were allowed" (Hammerl, 1990) 1 Unfortunately, we have not been able to find a proper reference to Guiraud's work of 1959. Therefore, we cannot tell if Guiraud was following some optimization hypothesis akin to optimal singular-coding or if he actually provided an exact formula like ours. Finally, the logarithmic relationship between the frequency of a word and its length in phonemes has also been inferred based on empirical data collected for overall eight languages (see Equation 11 in Guiter (1974)). However, this particular study is bare of any mathematical/information theoretic considerations.

Besides historical considerations, our findings also have practical implications for empirical research on the law of abbreviation as an indication of optimal coding. First, it is usually assumed that a significant negative correlation between frequency and magnitude is needed for efficient coding (Bezerra, Souto, Radford, \& Jones, 2011; Ferrer-i-Cancho \& Lusseau, 2009, Heesen et al., 2019; Semple et al., 2010). Our analyses indicate that a non-significant correlation can still be associated with efficient coding. For instance, we have seen that optimal coding with prescribed probabilities and magnitudes coming from some given multiset is equivalent to $\tau\left(p_{i}, l_{i}\right) \leq 0$ (Corollary 2.3). The same conclusion can be reached from optimal uniquely decodable coding, where all strings must have the same length when types are equally likely (recall Equation 9). Therefore, the influence of compression could be wider than commonly believed. What cannot be attributed to compression is the significant positive correlation between frequency and magnitude that has been found in a subset of the

\footnotetext{
${ }^{1}$ The German original reads "Guiraud (1959) hat aus rein kombinatorischen Überlegungen, wo alle möglichen Buchstabenkombinationen aus den Buchstaben der jeweiligen Sprache bei der Bildung von Wörtern zugelassen wurden [...] folgende Abhängigkeit [...] abgeleitet." This is followed by the formulae given above in the main text.
} 
repertoire of chimpanzee gestures, i.e. full body gestures (Heesen et al., 2019), in the vocalizations of female (but not male) hyraxes (Demartsev et al., 2019), phrases of male gibbon solos (Clink, Ahmad, \& Klinck, 2020), computer experiments with neural networks (Chaabouni et al. 2019) and also in European heraldry (Miton \& Morin, 2019). Importantly, this illustrates that compression - as reflected in the law of abbreviation - is not necessarily found in all communication systems, which undermines arguments that quantitative linguistic laws are unavoidable and hence "meaningless" (see also Ferrer-i-Cancho, Forns, Hernández-Fernández, Bel-Enguix, and Baixeries (2013) for the case of Menzerath's law).

Another argument along those lines is based on random typing: if random typing recreates Zipfian laws, then surely they are not an interesting subject of study (Miller, 1957). However, surprisingly, random typing turns out to be an optimal encoding system. Thus, finding linguistic laws in random typing does not preclude that these laws can be explained by information theoretic principles. However, while we have unveiled the optimality of random typing, we emphasize that we have done it only from the perspective of optimal non-singular coding. The fact that random typing and optimization are not independent issues as commonly believed (Chaabouni et al. 2019; Kanwal et al., 2017; Li, 1998; Miller, 1957), does not imply that random typing satisfies to a sufficient degree the optimization constraints imposed on natural languages.

We have seen that optimal non-singular coding predicts both a form of Zipf's law of abbreviation as well as a power-law distribution consistent with Zipf's law for word frequencies when combined with the maxent principle, revisiting an old argument by Mandelbrot (Mandelbrot, 1966). The capacity of maxent to obtain Zipfian laws as well as the less popular exponential distribution of parts-of-speech (Tuzzi et al., 2010) based on optimal and suboptimal coding considerations suggests that the principle should be considered as a critical component of a compact theory of linguistic patterns in general. For instance, $p(d)$, the probability that two syntactically related words are at distance $d$ (in words), exhibits an exponential decay that has been derived with the help of a combination of maxent and a constraint on the average value of $d$ (Ferrer-i-Cancho, 2004).

The principle of maximum entropy used to derive Zipf's law for word frequencies ensures that one is maximally uncertain about what one does not know (Kesavan, 2009). In the context of natural languages, a further justification of the use of the principle is that $I(S, R)$, the mutual information between words $(S)$ and meanings $(R)$ satisfies

$$
I(S, R) \leq H(S)
$$

where $H(S)$ is the entropy of words, namely the entropy of word probability ranks as defined above. The inequality in Equation 23 follows from elementary information theory (Cover \& Thomas, 2006), and has been applied to investigate the properties of dual optimization models of natural communication (Ferrer-i-Cancho \& Díaz-Guilera, $2007) . I(S, R)$ is a measure of the capacity of words to convey meaning: maximizing $I(S, R)$ one promotes that words behave like meaning identifiers (Ferrer-i-Cancho, 2018, Section 3). Therefore, Equation 23 suggests that the maximum entropy principle in the context of word entropy maximizes the potential of words to express meaning. The hypothesis of pressure to maximize $H(S)$ is supported by the skew towards the right that is found in the distribution of $H(S)$ in languages across the world (Bentz, Alikaniotis, Cysouw, \& Ferrer-i-Cancho, 2017). 
The challenge of mathematical modelling is to find a compromise between parsimony and predictive power (Burnham \& Anderson, 2002). Concerns about parsimony are a recurrent theme when modelling Zipf's law for word frequencies (Ferrer-i-Cancho, 2018, Mandelbrot, 1966; Visser, 2013). As for maximum entropy models, it has been argued that Shannon entropy and a logarithmic constraint offer the simplest explanation for the origins of the law (Visser, 2013). However, the argument is incomplete unless a justification for such a constraint is provided. Here we have shown how the logarithmic constraint follows from optimal non-singular coding. There are many possible explanations for the origins of Zipf's law based on maximum entropy (Baek et al. 2011; Ferrer-i-Cancho, 2005a; Liu, 2008; Mandelbrot, 1966; Naranan \& Balasubrahmanyan, 1992a, 1992b, 1993; Visser, 2013), and many more through other means (Mitzenmacher, 2003; Newman, 2005), but only compression can shed light on the origins of both Zipf's law for word frequencies and Zipf's law of abbreviation. The explanation of Zipf's law for word frequencies should not be separated from the explanation of other quantitative laws. Otherwise, the space of possible models is not sufficiently constrained (Stumpf \& Porter, 2012), and the resulting "theory" is not a well organized theory but a patchwork of models (Ferrer-i-Cancho, 2018).

Our theoretical framework is highly predictive in at least two senses. First, optimal coding predicts Zipf's law of abbreviation, but adherence to a traditional scheme (nonsingular coding or uniquely decodable coding) is not necessary. It suffices to assume that the magnitudes come from some predefined multiset. Second, its applicability goes beyond laws from Zipf's classic work. It can also be applied to Menzerath's law, the tendency of constructs with more parts to be made of smaller parts, i.e. the tendency of words with more syllables to be made of shorter syllables (Altmann, 1980). Taking the number of parts of constructs as probabilities of types $\left(p_{i}\right.$ 's $)$ and the size of the parts as magnitudes $\left(l_{i}\right.$ 's) and simply assuming that the number of parts are constant, Menzerath's law follows applying theorem 2.2 Gustison, Semple, Ferrer-i-Cancho, \& Bergman, 2016). This allows one to put forward optimization as a possible hypothesis to explain the pervasiveness of the law in nature (e.g. Boroda and Altmann (1991); Gustison et al. (2016); Shahzad, Mittenthal, and Caetano-Anollés (2015)).

\section{Acknowledgements}

This article is dedicated to the memory of P. Grzybek (1957-2019), co-author of one of the works referenced (Strauss et al., 2007). We thank A. Hernández-Fernández for many corrections and valuable comments and to L. Debowski for helping us to strengthen some of the mathematical proofs in the early stages of this research project. We also thank N. Ay and M. Gustison for helpful discussions. RFC is supported by the grant TIN2017-89244-R from MINECO (Ministerio de Economia, Industria y Competitividad) and the recognition 2017SGR-856 (MACDA) from AGAUR (Generalitat de Catalunya). CB is supported by the DFG Center for Advanced Studies Words, Bones, Genes, Tools at the University of Tübingen, and by the Swiss National Foundation Grant on "Non-randomness in Morphological Diversity: A Computational Approach Based on Multilingual Corpora" (SNF 176305) at the University of Zürich. CS is funded by a Melbourne Research Scholarship. 


\section{References}

Aadam. (2016). Minimum dot product. https://medium.com/competitive/minimum-dotproduct-62daa5281ba6.

Akmajian, A., Demers, R. A., Farmer, A. K., \& Harnish, R. M. (1995). Linguistics. an introduction to language and communication (4th ed.). MIT Press.

Altmann, G. (1980). Prolegomena to Menzerath's law. Glottometrika, 2, 1-10.

Baek, S. K., Bernhardsson, S., \& Minnhagen, P. (2011). Zipf's law unzipped. New Journal of Physics, 13(4), 043004.

Bentz, C., Alikaniotis, D., Cysouw, M., \& Ferrer-i-Cancho, R. (2017). The entropy of words - learnability and expressivity across more than 1000 languages. Entropy, 19(6).

Bentz, C., \& Ferrer-i-Cancho, R. (2016). Zipf's law of abbreviation as a language universal. In C. Bentz, G. Jäger, \& I. Yanovich (Eds.), Proceedings of the Leiden Workshop on Capturing Phylogenetic Algorithms for Linguistics. University of Tübingen.

Bezerra, B. M., Souto, A., Radford, A. N., \& Jones, G. (2011). Brevity is not always a virtue in primate communication. Biology letters, 7(1), 23-25.

Borda, M. (2011). Fundamentals in information theory and coding (1st ed.). Berlin: Springer.

Boroda, M. G., \& Altmann, G. (1991). Menzerath's law in musical texts. Musikometrika, 3, $1-13$.

Burnham, K. P., \& Anderson, D. R. (2002). Model selection and multimodel inference. A practical information-theoretic approach (2nd ed.). New York: Springer.

Casas, B., Català, N., Ferrer-i-Cancho, R., Hernández-Fernández, A., \& Baixeries, J. (2018). The polysemy of the words that children learn over time. Interaction Studies, 19, $389-$ 426.

Chaabouni, R., Kharitonov, E., Dupoux, E., \& Baroni, M. (2019). Anti-efficient encoding in emergent communication. arXiv:1905.12561.

Clink, D. J., Ahmad, A. H., \& Klinck, H. (2020). Brevity is not a universal in animal communication: evidence for compression depends on the unit of analysis in small ape vocalizations. Royal Society Open Science, 7(4), 200151.

Conover, W. J. (1999). Practical nonparametric statistics. New York: Wiley. (3rd edition)

Cover, T. M., \& Thomas, J. A. (2006). Elements of information theory. New York: Wiley. (2nd edition)

Demartsev, V., Gordon, N., Barocas, A., Bar-Ziv, E., T. Ilany, Y. G., Ilany, A., \& Geffen, E. (2019). The "Law of Brevity" in animal communication: Sex-specific signaling optimization is determined by call amplitude rather than duration. Evolution Letters, 3, 623-634.

Elias, P. (1975). Universal codeword sets and representations of the integers. IEEE Transactions on Information Theory, 21(2), 194-203.

Favaro, L., Gamba, M., Cresta, E., Fumagalli, E., Bandoli, F., Pilenga, C., .. R Reby, D. (2020). Do penguins' vocal sequences conform to linguistic laws? Biology Letters, 16 (2), 20190589.

Ferrer-i-Cancho, R. (2004). Euclidean distance between syntactically linked words. Physical Review E, 70, 056135.

Ferrer-i-Cancho, R. (2005a). Decoding least effort and scaling in signal frequency distributions. Physica A, 345, 275-284.

Ferrer-i-Cancho, R. (2005b). The variation of Zipf's law in human language. European Physical Journal B, 44, 249-257.

Ferrer-i-Cancho, R. (2015). The placement of the head that minimizes online memory: a complex systems approach. Language Dynamics and Change, 5, 114-137.

Ferrer-i-Cancho, R. (2018). Optimization models of natural communication. Journal of Quantitative Linguistics, 25, 207-237.

Ferrer-i-Cancho, R., \& Díaz-Guilera, A. (2007). The global minima of the communicative energy of natural communication systems. Journal of Statistical Mechanics, P06009.

Ferrer-i-Cancho, R., \& Elvevåg, B. (2009). Random texts do not exhibit the real Zipf's-law-like rank distribution. PLoS ONE, 5(4), e9411.

Ferrer-i-Cancho, R., Forns, N., Hernández-Fernández, A., Bel-Enguix, G., \& Baixeries, J. 
(2013). The challenges of statistical patterns of language: the case of Menzerath's law in genomes. Complexity, 18(3), 11-17.

Ferrer-i-Cancho, R., \& Gavaldà, R. (2009). The frequency spectrum of finite samples from the intermittent silence process. Journal of the American Association for Information Science and Technology, 60(4), 837-843.

Ferrer-i-Cancho, R., \& Hernández-Fernández, A. (2013). The failure of the law of brevity in two New World primates. Statistical caveats. Glottotheory, 4(1).

Ferrer-i-Cancho, R., Hernández-Fernández, A., Lusseau, D., Agoramoorthy, G., Hsu, M. J., \& Semple, S. (2013). Compression as a universal principle of animal behavior. Cognitive Science, 37(8), 1565-1578.

Ferrer-i-Cancho, R., \& Lusseau, D. (2009). Efficient coding in dolphin surface behavioral patterns. Complexity, $14(5), 23-25$.

Ficken, M. S., Hailman, J. P., \& Ficken, R. W. (1978). A model of repetitive behaviour illustrated by chickadee calling. Animal Behaviour, 26(2), 630-631.

Guiter, H. (1974). Les relationes frequence - longueur - sens des mots (langues romanes et anglais). In XIV Congresso Internazionale di linguistica e filologia romanza (p. 373-381). Napoli.

Gustison, M. L., Semple, S., Ferrer-i-Cancho, R., \& Bergman, T. (2016). Gelada vocal sequences follow Menzerath's linguistic law. Proceedings of the National Academy of Sciences USA, 113, E2750-E2758.

Hailman, J. P., Ficken, M. S., \& Ficken, R. W. (1985). The 'chick-a-dee' calls of Parus atricapillus: a recombinant system of animal communication compared with written English. Semiotica, 56, 121-224.

Hammerl, R. (1990). Länge - Frequenz, Länge - Rangnummer: Überprüfung von zwei lexikalischen Modellen. Glottometrika, 12, 1-24.

Harremoës, P., \& Topsøe, F. (2001). Maximum entropy fundamentals. Entropy, 3(3), 191226.

Heesen, R., Hobaiter, C., Ferrer-i-Cancho, R., \& Semple, S. (2019). Linguistic laws in chimpanzee gestural communication. Proceedings of the Royal Society B: Biological Sciences, 286, 20182900.

Huang, M., Ma, H., Ma, C., Garber, P. A., \& Fan, P. (2020). Male gibbon loud morning calls conform to Zipf's law of brevity and Menzerath's law: insights into the origin of human language. Animal Behaviour, 160, 145 - 155.

Kanwal, J., Smith, K., Culbertson, J., \& Kirby, S. (2017). Zipf's law of abbreviation and the principle of least effort: Language users optimise a miniature lexicon for efficient communication. Cognition, 165, 45-52.

Kapur, J. N., \& Kesavan, H. K. (1992). Entropy optimization principles and their applications. In V. P. Singh \& M. Fiorentino (Eds.), Entropy and energy dissipation in water resources (pp. 3-20). Dordrecht: Springer Netherlands.

Kesavan, H. K. (2009). Jaynes' maximum entropy principle. In C. A. Floudas \& P. M. Pardalos (Eds.), Encyclopedia of optimization (pp. 1779-1782). Boston, MA: Springer US.

Li, W. (1992). Random texts exhibit Zipf's-law-like word frequency distribution. IEEE T. Inform. Theory, 38(6), 1842-1845.

Li, W. (1998). Comments to "Zipf's law and the structure and evolution of languages" A.A. Tsonis, C. Schultz, P.A. Tsonis, Complexity, 2(5). 12-13 (1997). Complexity, 3, 9-10. (Letters to the editor)

Liu, C.-S. (2008). Maximal non-symmetric entropy leads naturally to Zipf's law. Fractals, 16(01), 99-101.

Luo, B., Jiang, T., Liu, Y., Wang, J., Lin, A., Wei, X., \& Feng, J. (2013). Brevity is prevalent in bat short-range communication. Journal of Comparative Physiology A, 199, 325-333.

Mandelbrot, B. (1966). Information theory and psycholinguistics: a theory of word frequencies. In P. F. Lazarsfield \& N. W. Henry (Eds.), Readings in mathematical social sciences (p. 151168). Cambridge: MIT Press.

McMillan, B. (1956). Two inequalities implied by unique decipherability. IRE Transactions 
on Information Theory, 2(4), 115-116.

Miller, G. A. (1957). Some effects of intermittent silence. Am. J. Psychol., 70, 311-314.

Miller, G. A., \& Chomsky, N. (1963). Finitary models of language users. In R. D. Luce, R. Bush, \& E. Galanter (Eds.), Handbook of mathematical psychology (Vol. 2, p. 419-491). New York: Wiley.

Miton, H., \& Morin, O. (2019). When iconicity stands in the way of abbreviation: No Zipfian effect for figurative signals. PLOS ONE, 14(8), 1-19.

Mitzenmacher, M. (2003). A brief history of generative models for power law and lognormal distributions. Internet Mathematics, 1, 226-251.

Moreno-Sánchez, I., Font-Clos, F., \& Corral, A. (2016). Large-scale analysis of Zipf's law in English. PLoS ONE, 11, 1-19.

Naranan, S., \& Balasubrahmanyan, V. K. (1992a). Information theoretic models in statistical linguistics - Part I: A model for word frequencies. Current Science, 63, 261-269.

Naranan, S., \& Balasubrahmanyan, V. K. (1992b). Information theoretic models in statistical linguistics - Part II: Word frequencies and hierarchical structure in language. Current Science, 63, 297-306.

Naranan, S., \& Balasubrahmanyan, V. K. (1993). Information theoretic model for frequency distribution of words and speech sounds (phonemes) in language. Journal of Scientific and Industrial Research, 52, 728-738.

Newman, M. E. J. (2005). Power laws, Pareto distributions and Zipf's law. Contemporary Physics, 46, 323-351.

Piantadosi, S. T., Tilly, H., \& Gibson, E. (2012). The communicative function of ambiguity in language. Cognition, 122(3), 280 - 291.

Ramscar, M. (2019). Source codes in human communication. https://psyarxiv.com/e3hps.

Rapoport, A. (1982). Zipf's law re-visited. In H. Guiter \& M. V. Arapov (Eds.), Quantitative linguistis: Studies on Zipf's law (p. 1-28). Bochum: Studienverlag Dr. N. Brockmeyer.

Romberg, A. R., \& Saffran, J. R. (2010). Statistical learning and language acquisition. Wiley Interdisciplinary Reviews: Cognitive Science, 1(6), 906-914.

Semple, S., Hsu, M. J., \& Agoramoorthy, G. (2010). Efficiency of coding in macaque vocal communication. Biology Letters, 6, 469-471.

Shahzad, K., Mittenthal, J., \& Caetano-Anollés, G. (2015). The organization of domains in proteins obeys Menzerath-Altmann's law of language. BMC Systems Biology, 9, 1-13.

Sigurd, B., Eeg-Olofsson, M., \& van Weijer, J. (2004). Word length, sentence length and frequency - Zipf revisited. Studia Linguistica, 58(1), 37-52.

Strauss, U., Grzybek, P., \& Altmann, G. (2007). Word length and word frequency. In P. Grzybek (Ed.), Contributions to the science of text and language (p. 277-294). Dordrecht: Springer.

Stumpf, M. P. H., \& Porter, M. A. (2012). Critical truths about power laws. Science, $335(6069), 665-666$.

Sudan, M. (2006). Lecture 07 (03/02): Source coding: Shannon codes, huffman codes. transmission of information. http://people.csail.mit.edu/madhu/ST06/scribe/ LO7_xshi_main.pdf.

Suzuki, R., Tyack, P. L., \& Buck, J. (2005). The use of Zipf's law in animal communication analysis. Anim. Behav., 69, 9-17.

Tuzzi, A., Popescu, I.-I., \& Altmann, G. (2010). Quantitative analysis of Italian texts (Vol. 6). Lüdenscheid, Germany: RAM Verlag.

Visser, M. (2013). Zipf's law, power laws and maximum entropy. New Journal of Physics, $15(4), 043021$.

Zipf, G. K. (1949). Human behaviour and the principle of least effort. Cambridge (MA), USA: Addison-Wesley. 